\title{
Quantum Reflection of Antihydrogen from Nanoporous Media
}

\author{
G. Dufour, ${ }^{1}$ R. Guérout,${ }^{1}$ A. Lambrecht,${ }^{1}$ V.V. Nesvizhevsky, ${ }^{2}$ S. Reynaud,${ }^{1}$ and A.Yu. Voronin ${ }^{3}$ \\ ${ }^{1}$ Laboratoire Kastler-Brossel, CNRS, ENS, UPMC, Campus Jussieu, F-75252 Paris, France \\ ${ }^{2}$ Institut Laue-Langevin (ILL), 6 rue Jules Horowitz, F-38042, Grenoble, France \\ ${ }^{3}$ P.N. Lebedev Physical Institute, 53 Leninsky prospect, Ru-117924 Moscow, Russia
}

\begin{abstract}
We study quantum reflection of antihydrogen atoms from nanoporous media due to the CasimirPolder (CP) potential. Using a simple effective medium model, we show a dramatic increase of the probability of quantum reflection of antihydrogen atoms if the porosity of the medium increases. We discuss the limiting case of reflections at small energies, which have interesting applications for trapping and guiding antihydrogen using material walls.
\end{abstract}

PACS numbers: $36.10 . \mathrm{Gv}, 34.35 .+\mathrm{a}, 12.20 \mathrm{Ds}$

\section{INTRODUCTION}

In a previous paper 1 we have shown that antihydrogen atoms have a significant probability of being reflected when they fall on a material surface. This quantum reflection occurs as a consequence of the rapid variation of the Casimir-Polder (CP) interaction on the scale of the atomic de Broglie wavelength 2 12.

We did emphasize in 1 that quantum reflection increased for weaker CP potentials. The explanation is that quantum reflection takes place closer to the surface for weaker potentials, and that the potential is steeper there, leading to a greater probability of non-adiabatic transition. As the CP interaction is due to the coupling of atoms with surface through electromagnetic vacuum fluctuations, a weaker coupling of the surface to the electromagnetic field leads to increased quantum reflection. This paradoxical result was discussed in [1] in relation to the effect of the dielectric index, as well as that of thickness for matter slabs. Here, we wish to extend this work by focusing on the enhancement of quantum reflection from nanoporous materials.

Increasing the reflection probability of antihydrogen would open the possibility for trapping and guiding antimatter with material walls, in particular anti-atoms can be trapped in gravitational quantum states held by gravity from above and quantum reflection from below [13, 14. Such states are also of interest for the GBAR experiment which is designed to measure the gravitational properties of antimatter [15]. In the current design, the acceleration of gravity $\bar{g}$ for antihydrogen atoms is measured by timing their fall from the release from a trap to the annihilation at contact with a matter plate. In the future, the acceleration of gravity $\bar{g}$ could be measured with a better precision through a spectroscopic study of gravitational quantum states.

In the present paper, we will consider several examples of nanoporous media : silica aerogels, porous silicon and powders of diamond nanoparticles. These materials incorporate a significant fraction of gas or vacuum, which reduces their effective permittivity. We expect a significant increase in quantum reflection probability when the average density of the medium is significantly smaller than the bulk density of the constituent material. As inhomogeneities in such materials have a nanometric scale, we may describe the material by an effective medium theory and still expect approximately correct results for processes involving larger scales.

Silica aerogels are generally produced by supercritically drying a silica gel, a process which removes the liquid component while leaving the silica matrix undamaged. The result is a disordered array of pores in a solid framework whose properties such as porosity (volume fraction of gas or vacuum) and pore size are tunable. Porosities as high as $98 \%$ are obtained for aerogels, with pore sizes typically of the order of 20-100 nm [16]. Porous silicon can be obtained by anodization or etching of a silicon wafer. Porosities up to $95 \%$ are obtained with pore sizes typically ranging from 2 to $50 \mathrm{~nm}[17,18$.

Diamond nanoparticles formed by explosive shock have been observed for more than forty years 19. Intensive studies of their fabrication and applications have been performed worldwide [20]. These particles, with typical sizes of a few nanometers, consist of a diamond nucleus (with diamond density) within a thin onion-like shell with complex chemical composition and lower density 21. Powders of diamond nanoparticles have been intensively studied and used in neutron investigations in relation to their unique property of extremely efficient reflection of slow neutrons 22,23 . The analogy between the interaction of slow antihydrogen atoms and neutrons with nanodiamond powders (via the $\mathrm{CP}$ and Fermi interactions respectively) is underlined by the fact that their masses, typical velocities and wavelengths are equal. The typical density in powder varies between $5 \%$ and $15 \%$ of the diamond density, depending on the conditioning of the powder; it can be increased by shaking or pressing the powder, and decreased by blowing air through it. The density close to surface of a nanoparticle powder sample is even lower than that the bulk density of the powder (see for instance [24]).

Quantum reflection experiments have been performed by Pasquini et al with condensates of sodium atoms on a nano-structured silicon surface and a silica aerogel [10. A reflection probability of $\sim 60 \%$ was observed on the nano-structured silicon surface, compared with $\sim 15 \%$ 
on bulk silicon. However, no reflection was observed on the aerogel. The authors of [10 impute this observation to uncontrolled surface charges. This is a serious issue to be solved in order to use the spectacular enhancement of reflection predicted in the present paper.

The dielectric properties of the material over a broad range of frequencies are needed to compute the $\mathrm{CP}$ force. Though a porous medium is by nature inhomogeneous, we will use an effective medium approximation and describe the composite material as an homogeneous medium with an effective permittivity $\epsilon$. We will also make the simplifying assumption of a plane interface, whereas a porous medium is likely to have a rough surface. Such approximations are valid if the wavelengths involved are larger than the scales of inhomogeneity or roughness. Qualitatively, one expects our simplified description to lead to approximately correct results if the atom does not approach the medium closer than these scales. It will therefore be sufficient for a first exploration of the enhancement of quantum reflection by the use of low-density materials. A more complete description will require further work, as it would have to study the $\mathrm{CP}$ effect in presence of non specular scattering of electromagnetic field 25] as well as the resulting non specular quantum reflection of antihydrogen.

\section{EFFECTIVE MEDIUM MODEL}

There are several models available for obtaining the effective permittivity $\epsilon$ as a function of the permittivities of the constituent materials. For a host material containing non-overlapping spherical inclusions of another material, application of the Clausius-Mossoti relation yields the Maxwell-Garnett formula [26]:

$$
\frac{\epsilon-\epsilon_{h}}{\epsilon+2 \epsilon_{h}}=\phi_{i} \frac{\epsilon_{i}-\epsilon_{h}}{\epsilon_{i}+2 \epsilon_{h}}
$$

where $\epsilon_{h}$ and $\epsilon_{i}$ are the permittivity of the host and inclusions respectively and $\phi_{i}$ is the volume fraction of inclusions.

Another possibility is the Bruggeman model [27, which requires that the average polarization of spherical inclusions embedded in the effective medium vanish. For a mixture of materials with permittivities $\epsilon_{1}$ and $\epsilon_{2}$ and volume fractions $\phi_{1}$ and $\phi_{2}\left(\phi_{1}+\phi_{2}=1\right)$, the effective permittivity $\epsilon$ is given by:

$$
\phi_{1} \frac{\epsilon_{1}-\epsilon}{\epsilon_{1}+2 \epsilon}+\phi_{2} \frac{\epsilon_{2}-\epsilon}{\epsilon_{2}+2 \epsilon}=0
$$

Landau and Lifshitz have proposed another effective model based on the idea that the cubic root of the permittivity is approximately additive [28]:

$$
\epsilon^{1 / 3}=\phi_{1} \epsilon_{1}^{1 / 3}+\phi_{2} \epsilon_{2}^{1 / 3}
$$

In the Casimir-related literature, aerogels have been described by Maxwell-Garnett models with air inclusions in a solid matrix [29] or vice-versa [30]. Note that this Maxwell-Garnett model does not treat the host and the inclusions in a symmetrical manner. Here we prefer to use a symmetrical model, like the Landau-Lifshitz and Bruggeman models, since we want to vary the porosity over a wide range of values. As shown in figure 1, the Landau-Lifshitz and Bruggeman models give very similar results and we chose the latter for its simpler interpretation. In the rest of this paper, we denote $\phi$ the porosity, i.e. the volume fraction of gas or vacuum, and $1-\phi$ the solid fraction, that is also the density reduction.

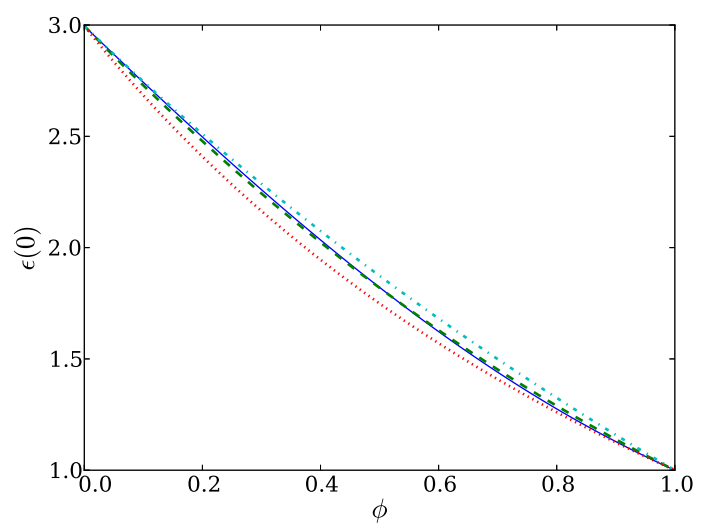

FIG. 1. (Color online) Comparison of effective medium models for the effective static permittivity of a silica aerogel as a function of porosity: Bruggeman (blue full line), LandauLifschitz (green dashed line), Maxwell-Garnett with air inclusions (cyan dot-dashed line), Maxwell-Garnett with silica inclusions (red dotted line).

The optical response properties used for antihydrogen, silica and silicon are the same as in [1. Diamond is described by a simple Sellmeier model [31]:

$$
\epsilon(i \xi)=B_{0}+\frac{B_{1}}{1+\left(\xi / \omega_{1}\right)^{2}}+\frac{B_{2}}{1+\left(\xi / \omega_{2}\right)^{2}},
$$

with the parameters $B_{0,1,2}=2.30982863,3.35656148$, 3.25669602 and $\omega_{1,2}=14.3235,0.0376730 \times 10^{15} \mathrm{rad} . \mathrm{s}^{-1}$.

\section{CASIMIR-POLDER POTENTIAL}

The calculation of the $\mathrm{CP}$ potential, presented in [1, is not repeated here. Results are presented with reference to the long-range retarded potential calculated for a perfect mirror $V^{*}(z)=C_{4}^{*} / z^{4}$ with $C_{4}^{*}=2.5 \times 10^{-57} \mathrm{Jm}^{4}=73.6$ a.u. . Figures 2 and 3 show the CP potential created by aerogels and powders of diamond nanoparticles respectively, for various values of the porosity. The potential curves for porous silicon are similar to those of diamond nanoparticles. Figure 4 compares the potentials of these three materials when the porosity is $0 \%$ and $90 \%$. 


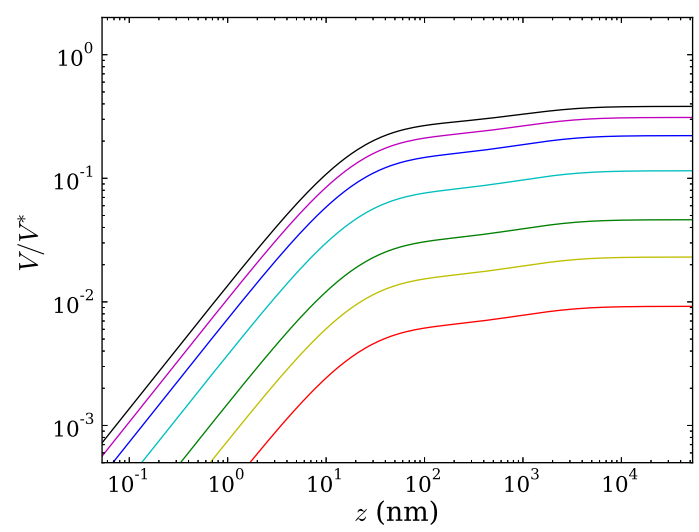

FIG. 2. (Color online) CP potential $V$ for antihydrogen on silica aerogels, divided by the long-range potential $V^{*}$ for a perfect mirror. From top to bottom the porosity is $0 \%$ (bulk silica, black), 25\% (magenta), $50 \%$ (blue), $75 \%$ (cyan), $90 \%$ (green), 95\% (yellow), 98\% (red).

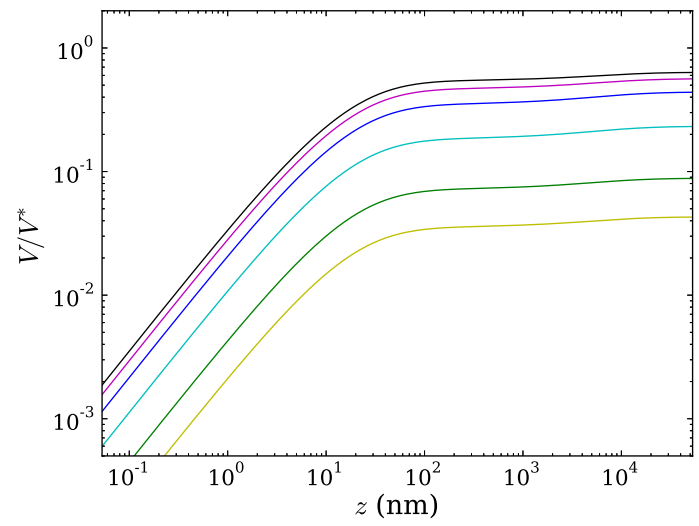

FIG. 3. (Color online) CP potential $V$ for antihydrogen on a powder of nano-diamonds, divided by the long-range potential $V^{*}$ for a perfect mirror. From top to bottom the porosity is 0\% (bulk diamond, black), 25\% (magenta), $50 \%$ (blue), $75 \%$ (cyan), 90\% (green), 95\% (yellow).

As explained in 1, quantum reflection occurs in the so-called badlands regions where the adiabatic approximation breaks down. Nonadiabatic transitions are effective when the function $Q(z)=\hbar^{2} p^{\prime \prime}(z) / 2 p(z)^{3}-3 \hbar^{2} p^{\prime}(z)^{2} / 4 p(z)^{4}$ is large. It is expressed here as a function of the classical momentum $p=\sqrt{2 m(E-V(z))}$ and is related to the Schwarzian derivative of the WKB phase (see the references in [1]).

The peak of this function gives an indication on the distance where the reflection occurs and on the magnitude of the reflection. The peak is located closer to the surface for a weaker potential, thus corresponding to a steeper variation and a larger $Q$. This is illustrated in figure 5 for silica aerogels. When porosity is increased,

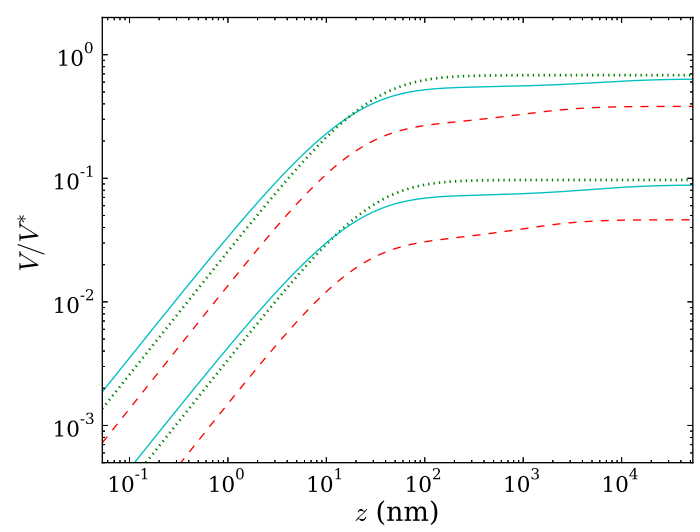

FIG. 4. (Color online) CP potential $V$ for antihydrogen on silica (red dashed line), silicon (green dotted line) diamond (cyan full line), divided by the long-range potential $V^{*}$ for a perfect mirror. The top three curves are for bulk materials, the bottom three for $90 \%$ porosity.

the peak of the badlands function $Q$ gets closer to the surface while its magnitude grows.

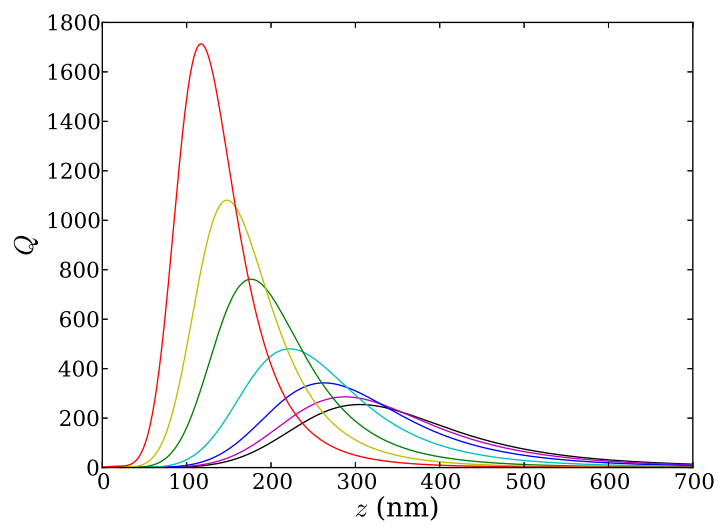

FIG. 5. (Color online) Badlands function $Q$ for antihydrogen approaching silica aerogels with velocity $v=10 \mathrm{~mm} \cdot \mathrm{s}^{-1}$. From bottom right to top left the porosity is 0\% (bulk silica, black), 25\% (magenta), 50\% (blue), 75\% (cyan), 90\% (green), 95\% (yellow), $98 \%$ (red).

We note that reflection occurs at a distance $\gtrsim 100 \mathrm{~nm}$ if the velocity of atoms is kept below $10 \mathrm{~mm} \cdot \mathrm{s}^{-1}$, even for the poorest reflective material considered here (silica aerogel with $98 \%$ porosity). We therefore except that our simplified model gives qualitatively correct results as long as the condition on velocity is obeyed. This is the case for the regime of large quantum reflections obtained at low energies. 


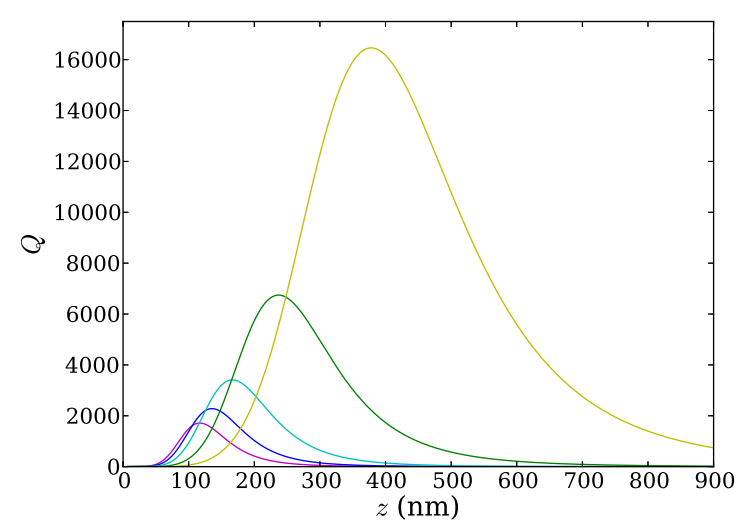

FIG. 6. (Color online) Badlands function $Q$ for antihydrogen approaching $98 \%$ porosity silica aerogel. From bottom left to top right the atom's velocity is $10 \mathrm{~mm} . \mathrm{s}^{-1}$ (magenta), 7.5 mm.s ${ }^{-1}$ (blue), $5.0 \mathrm{~mm} . \mathrm{s}^{-1}$ (cyan), $2.5 \mathrm{~mm} . \mathrm{s}^{-1}$ (green), 1.0 $\mathrm{mm} \cdot \mathrm{s}^{-1}$ (yellow).

\section{SCATTERING LENGTH}

We use the method described in [1] to calculate the reflection probability of antihydrogen atoms on the medium. In particular we enforce a full absorption boundary condition on the surface to account for the annihilation of those antihydrogen atoms which come to contact with matter. We focus the discussion on the low-energy behavior of atoms which corresponds to large quantum reflection.

The reflection amplitude $r$ can be written in terms of a complex scattering length $a$, which is defined as in [1]. For ultra-cold antihydrogen atoms, that is for velocities below $10 \mathrm{~mm} \cdot \mathrm{s}^{-1}, a$ is independent of the energy. Its real and imaginary parts are shown in figures 7 and 8 respectively, for varying porosities.

When considering quantum gravitational traps for $\overline{\mathrm{H}}$ bounded below by the quantum reflection from the $\mathrm{CP}$ potential and above by gravity [13, 14, one obtains the lifetime for the quantum bouncer in the first gravitational quantum state:

$$
\tau=\frac{\hbar}{2 m g|\operatorname{Im} a(0)|}
$$

Figure 9 shows this lifetime for silica aerogel, porous silicon and powder of diamond nanoparticles as the porosity is varied. The $y$ - axis starts at $0.1 \mathrm{~s}$ which is the lifetime calculated for a perfect mirror 13. Reflection is dramatically enhanced, especially for silica aerogels, for which extremely high porosities can be reached. For porous silicon and diamond nanoparticles, the reflection is enhanced by a factor $\sim 6$ between $0 \%$ and $95 \%$ porosity whereas for silica aerogel the enhancement factor is more than 20 between $0 \%$ and $98 \%$ porosity, with a lifetime reaching $4.6 \mathrm{~s}$ for the latter.

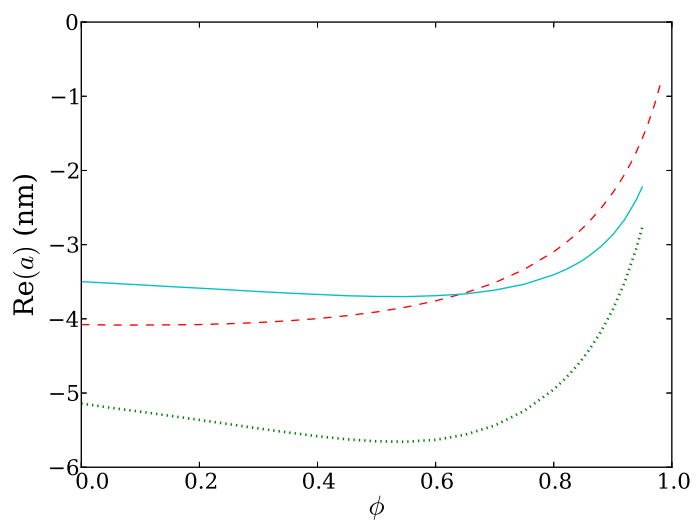

FIG. 7. (Color online) Real part of the scattering length $a$ as a function of porosity $\phi$ for antihydrogen on a silica aerogel (red dashed line), porous silicon (green dotted line) and a powder of nanodiamonds (cyan full line).

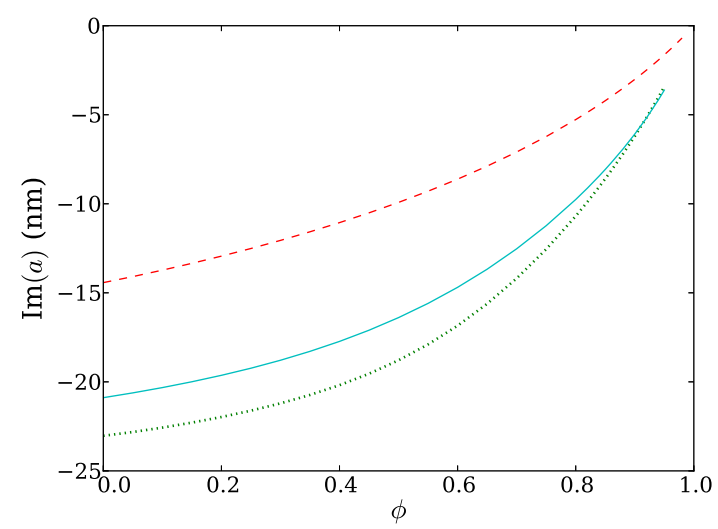

FIG. 8. (Color online) Imaginary part of the scattering length $a$ as a function of porosity $\phi$ for antihydrogen on a silica aerogel (red dashed line), porous silicon (green dotted line) and a powder of nanodiamonds (cyan full line).

\section{CONCLUSION}

Using a simple effective medium model, we have shown a dramatic increase of the quantum reflection probability of antihydrogen atoms from nanoporous media. We have given theoretical predictions for reflection on silica aerogels, porous silicon and powders of diamond nanoparticles over a wide range of porosities. These results open exciting perspectives for trapping antihydrogen atoms above material surfaces and investigating its gravitational properties, although more work is needed to quantify the effects of inhomogeneities in the nanoporous medium. 


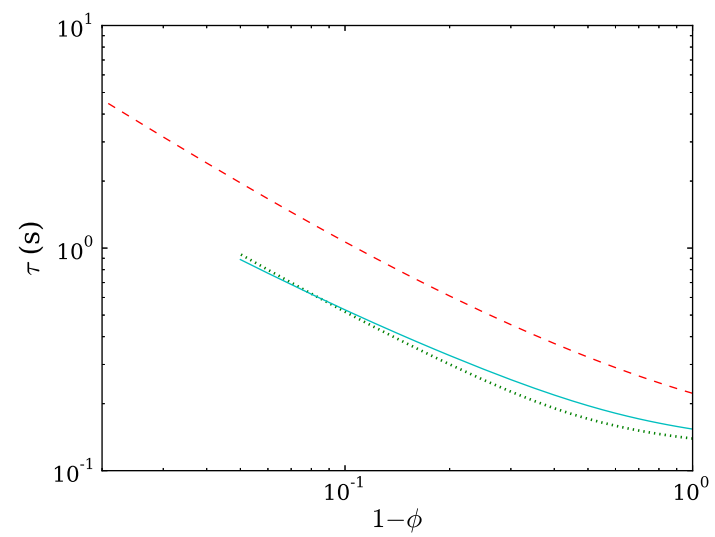

FIG. 9. (Color online) Lifetime $\tau$ for antihydrogen in the first gravitational state above a silica aerogel (red dashed line), porous silicon (green dotted line) and a powder of nanodiamonds (cyan full line), as a function of the solid fraction $1-\phi$ $(\log -\log$ scale).

[1] G. Dufour, A. Gérardin, R. Guérout, A. Lambrecht, V. Nesvizhevsky, S. Reynaud, and A. Voronin, Physical Review A, accepted (2012).

[2] M. Berry and K. Mount, Reports on Progress in Physics, 35, 315 (1972)

[3] I. Yu, J. Doyle, J. Sandberg, C. Cesar, D. Kleppner, and T. Greytak, Phys. Rev. Lett., 71, 1589 (1993).

[4] F. Shimizu, Phys. Rev. Lett., 86, 987 (2001).

[5] V. Druzhinina and M. DeKieviet, Phys. Rev. Lett., 91, $193202(2003)$

[6] H. Oberst, Y. Tashiro, K. Shimizu, and F. Shimizu, Phys. Rev., A71, 052901 (2005)

[7] V. P. A. Lonij, W. F. Holmgren, and A. D. Cronin, Phys. Rev. A, 80, 062904 (2009)

[8] B. S. Zhao, H. C. Schewe, G. Meijer, and W. Schoellkopf, Phys. Rev. Lett., 105, 133203 (2010).

[9] T. Pasquini, Y. Shin, C. Sanner, M. Saba, A. Schirotzek, D. Pritchard, and W. Ketterle, Phys. Rev. Lett., 93, 223201 (2004).

[10] T. A. Pasquini, M. Saba, G.-B. Jo, Y. Shin, W. Ketterle, D. E. Pritchard, T. A. Savas, and N. Mulders, Phys. Rev. Lett., 97, 093201 (2006).

[11] H. Friedrich, G. Jacoby, and C. Meister, Physical Review A, 65, 032902 (2002).

[12] T. Judd, R. Scott, A. Martin, B. Kaczmarek, and T. Fromhold, New Journal of Physics, 13, 083020 (2011).

[13] A. Voronin, P. Froelich, and V. Nesvizhevsky, Physical Review A, 83, 032903 (2011).

[14] A. Y. Voronin, V. V. Nesvizhevsky, and S. Reynaud, Journal of Physics B, 45 (2012), ISSN 0953-4075, doi: 10.1088/0953-4075

[15] G. Chardin, P. Grandemange, D. Lunney, V. Manea, A. Badertscher, P. Crivelli, A. Curioni, A. Marchionni, B. Rossi, A. Rubbia, V. Nesvizhevsky, P.-A. Hervieux, G. Manfredi, P. Comini, P. Debu, P. Dupré, L. Liszkay, B. Mansoulié, P. Pérez, J.-M. Rey, N. Ruiz, Y. Sac- quin, A. Voronin, F. Biraben, P. Cladé, A. Douillet, A. Gérardin, S. Guellati, L. Hilico, P. Indelicato, A. Lambrecht, R. Guérout, J.-P. Karr, F. Nez, S. Reynaud, V.Q. Tran, A. Mohri, Y. Yamazaki, M. Charlton, S. Eriksson, N. Madsen, D.-P. van der Werf, N. Kuroda, H. Torii, and Y. Nagashima, Proposal to measure the Gravitational Behaviour of Antihydrogen at Rest, Tech. Rep. CERNSPSC-2011-029. SPSC-P-342 (CERN, Geneva, 2011).

[16] K. Sinko, Materials, 3, 704 (2010), ISSN 1996-1944.

[17] P. Granitzer and K. Rumpf, Materials, 3, 943 (2010), ISSN 1996-1944.

[18] O. Bisi, S. Ossicini, and L. Pavesi, Surface Science Reports, 38, 1 (2000), ISSN 0167-5729.

[19] P. Decarli and J. Jamieson, Science, 133, 1821 (1961), ISSN 0036-8075.

[20] V. Y. Dolmatov, Russian Chemical Reviews, 76, 339 (2007), ISSN 0036-021X, 1468-4837.

[21] A. E. Aleksenskii, M. V. Baidakova, A. Y. Vul', and V. I. Siklitskii, Physics of the Solid State, 41, 668 (1999), ISSN 1063-7834.

[22] V. V. Nesvizhevsky, E. V. Lychagin, A. Y. Muzychka, A. V. Strelkov, G. Pignol, and K. V. Protasov, Nuclear Instruments \& Methods A, 595, 631 (2008), ISSN 01689002, WOS:000261028600012.

[23] V. Nesvizhevsky, R. Cubitt, E. Lychagin, A. Muzychka, G. Nekhaev, G. Pignol, K. Protasov, and A. Strelkov, Materials, 3, 1768 (2010), ISSN 1996-1944, WOS:000298240500014.

[24] V. V. Nesvizhevsky, A. Y. Voronin, A. Lambrecht, and S. Reynaud, New Journal of Physics, 14 (2012), ISSN 1367-2630.

[25] A. M. Contreras-Reyes, R. Guerout, P. A. M. Neto, D. A. R. Dalvit, A. Lambrecht, and S. Reynaud, Physical Review A, 82, 052517 (2010).

[26] J. C. Maxwell Garnett, Philos T R Soc A, 203, 385 (1904) 
[27] D. A. G. Bruggeman, Annalen der Physik, 416, 636 (1935), ISSN 1521-3889.

[28] L. Landau and E. Lifshitz, Electrodynamics of continuous media, Course of Theoretical Physics (Pergamon Press, 1960).
[29] R. Esquivel-Sirvent, Journal of Applied Physics, 102, 034307 (2007).

[30] G. Bimonte, T. Emig, M. Krüger, and M. Kardar, Physical Review A, 84, 042503 (2011).

[31] G. Ghosh, Handbook of thermo-optic coefficients of optical materials with applications (Academic Press, San Diego, Etats-Unis, 1998) ISBN 0-12-281855-5. 